\title{
A MEMOIR ON THE DOCTRINE OF ASSOCIATED FORMS*
}

\author{
BY
}

\section{O. E. GLENN}

The theory of associated forms, founded by Hermite, $\uparrow$ and developed by Clebsch, and Sylvester, and by many others, has as its primary object the study of sets of concomitants of a system of forms $F_{1}, F_{2}, \cdots$, such that every concomitant of the system is quasi-reducible, on multiplication by a power of a certain concomitant of a set, in terms of the members of this set. By expansion of each form of a system of binary forms, in terms of a pair of linear covariants as arguments, there is obtained the so-called typical representation of the system in which all coefficients are invariants; and every concomitant of the system is rational in the associated set consisting of the totality of these invariants, the two argument forms, and a multiplier which is the resultant of the two arguments. Extension to typical representation of binary forms of even order in terms of three quadratic covariants as arguments has been made by Clebsch. $\ddagger$

In the first section of the present paper is given a generalization of the above theory, in which the system of ground forms is represented typically in terms of two quadratic covariants, and, under restrictions, in terms of two nics. This generalization is both natural and comprehensive, and gives remarkable syzygetic relations among the forms of a compiete system.

The second section is devoted to associated and to complete systems of concomitants of a binary form $f$ under a general linear transformation $t$, which are functions of the coefficients of $t$ as well as of the coefficients and variables of $f$. These concomitants have various unique properties; for instance, the factor $r$ of a concomitant $C$ in the equation expressing the invariant property, $C^{\prime}=r C$, is not, in all cases, a power of the determinant of $t$.

In the third section one of a system of ground forms is assumed to be a universal covariant of a special group. If the usual domain of rationality of concomitants is then altered by adjunction of the multiplier invariant of a certain associated set (an absolute constant), this set furnishes a fundamental system under the special group. Certain obscurities in the theory of in-

* Presented to the Society, under different titles, October 28, 1916, and December 27, 1916.

† Hermite, Journal f ür Mathematik, vol. 52 (1856), p. 1.

$\ddagger$ Theorie der binären-algebraischen Formen (1872), p. 410. 
variants and covariants of special transformations, which have persisted since the time of Boole, are made clear by thus identifying their theory with the general doctrine of associated forms.

\section{Generalizations in the theORY OF ASSOCIATED FORMS}

1. Formal groundwork of the theory. I showed a few years since that the binary form $f$ of order $n(m+1)-1$, whose coefficients are arbitrary variables, has a unique expansion* in terms of any two binary nics $f_{1 n}, f_{2 n}$,

$$
f_{1 n}=\alpha_{0} x_{1}^{n}+n \alpha_{1} x_{1}^{n-1} x_{2}+\cdots, \quad f_{2 n}=\beta_{0} x_{1}^{n}+n \beta_{1} x_{1}^{n-1} x_{2}+\cdots,
$$

provided only that the resultant $R$ of $f_{1 n}, f_{2 n}$ does not vanish, in the form

$$
f=\sum_{i=0}^{m}\left(\begin{array}{c}
m \\
i
\end{array}\right) \phi_{i n-1} f_{1 n}^{m-i} f_{2 n}^{i}
$$

The coefficients $\phi_{i n-1}(i=0, \cdots, m)$ were determined as binary quantics of order $n-1$ in $x_{1}, x_{2}$, with coefficients linear in the coefficients $a_{0}, \cdots$, $a_{n(m+1)-1}$ of $f$ and rational in the quantities $\alpha_{0}, \cdots, \alpha_{n}, \beta_{0}, \cdots, \beta_{n}$; integral also in the latter, save for a common denominator $R^{m}$. These conclusions corformed to the theory of a symbolical basis of a finite expansion, according to which $f$ is represented symbolically as the $m$ th power

$$
f=\left(\Xi_{1} f_{1 n}+\Xi_{2} f_{2 n}\right)^{m}=\Xi_{f_{n}}^{m}=\Xi_{f_{n}}^{\prime m},
$$

and the quantics $\phi_{i n-1}=\Xi_{1}^{m-i} \Xi_{2}^{i}(i=0, \cdots, m)$ are all of the same type, in the sense of covariant types; satisfying the equations

$$
\begin{aligned}
& -\left(\alpha \frac{\partial}{\partial \beta}\right) \phi_{i n-1}=(m-i) \phi_{i+1 n-1} \\
& -\left(\beta \frac{\partial}{\partial \alpha}\right) \phi_{i n-1}=i \phi_{i-1 n-1} ; \quad\left(\beta \frac{\partial}{\partial \alpha}\right)=\beta_{0} \frac{\partial}{\partial \alpha_{0}}+\cdots+\beta_{n} \frac{\partial}{\partial \alpha_{n}} .
\end{aligned}
$$

The infinitude of symbolical expressions

$$
F=\sum k\left(\Xi^{\prime} \Xi^{\prime}\right)^{p}\left(\Xi^{\Xi^{\prime \prime}}\right)^{q} \cdots \Xi_{f_{n}}^{s} \Xi_{f_{n}}^{\prime t} \cdots,
$$

constructed by analogy with the symbolical concomitants of a binary mic, represent rational and integral isobaric functions of the forms $\phi_{i n-1}, f_{1 n}, f_{2 n}$, and all $F$ 's are annihilated by the three isomorphic operators

$$
\Xi_{2} \frac{\partial}{\partial \Xi_{1}}-f_{1 n} \frac{\partial}{\partial f_{2 n}}
$$

(b) $m \phi_{1 n-1} \frac{\partial}{\partial \phi_{0 n-1}}+(m-1) \phi_{2 n-1} \frac{\partial}{\partial \phi_{1 n-1}}+\cdots+\phi_{m n-1} \frac{\partial}{\partial \phi_{m-1 n-1}}-f_{1 n} \frac{\partial}{\partial f_{2 n}}$,

* These Transactions, vol. 15 (1914), p. 72. 


$$
-\left(\alpha_{0} \frac{\partial}{\partial \beta_{0}}+\alpha_{1} \frac{\partial}{\partial \beta_{1}}+\cdots+\alpha_{n} \frac{\partial}{\partial \beta_{n}}\right),
$$

and therefore by $[\beta(\partial / \partial \alpha)]$ also.* This is a sufficient as well as a necessary condition for the invariance of the $F$ 's under the formal alterations of the quantics $f_{1 n}, f_{2 n}$, given by the equalities

$$
f_{1 n}^{\prime}=\lambda_{1} f_{1 n}+\mu_{1} f_{2 n}, \quad f_{2 n}^{\prime}=\lambda_{2} f_{1 n}+\mu_{2} f_{2 n},
$$

where $(\lambda \mu) \neq 0 ; f_{1 n}^{\prime}=\alpha_{0}^{\prime} x_{1}^{n}+n \alpha_{1}^{\prime} x_{1}^{n-1} x_{2}+\cdots$.

In this paper much use is made of the explicit expressions for the forms $\phi_{\text {in }-1}$ in terms of the $\alpha$ 's and $\beta$ 's. If we assume

$$
\phi_{i n-1}=p_{i 0} x_{1}^{n-1}+p_{i 1} x_{1}^{n-2} x_{2}+\cdots+p_{i n-1} x_{2}^{n-1} \quad(i=0, \cdots, m),
$$

and regard equation (1) as an identity, it gives just a sufficient number of linear equations in the quantities $p_{i j}$ for their unique determination by Cramer's rule. The absolute terms of this linear system are respectively the coefficients $a_{0}, a_{1}, \cdots, a_{n(m+1)-1}$ of $f$. The matrix of the system is the matrix of the coefficients of the $n(m+1)$ forms of order $n(m+1)-1$ below, save for binomial multipliers common to elements of columns, which we omit (cf. (1)):

$$
\begin{aligned}
x_{1}^{i} x_{2}^{j} f_{1 n}^{m}, x_{1}^{i} x_{2}^{j} f_{1 n}^{m-1} f_{2 n}, & \cdots, x_{1}^{i} x_{2}^{j} f_{2 n}^{m} \\
& (j=0, \cdots, n-1 ; i=n-j-1) .
\end{aligned}
$$

The determinant of this matrix is a constant times $R^{\frac{1}{m(m+1)}} \neq 0$.

Example. If $n=2$ and $f$ is any form of odd order $2 m+1$, the matrix is

(2)

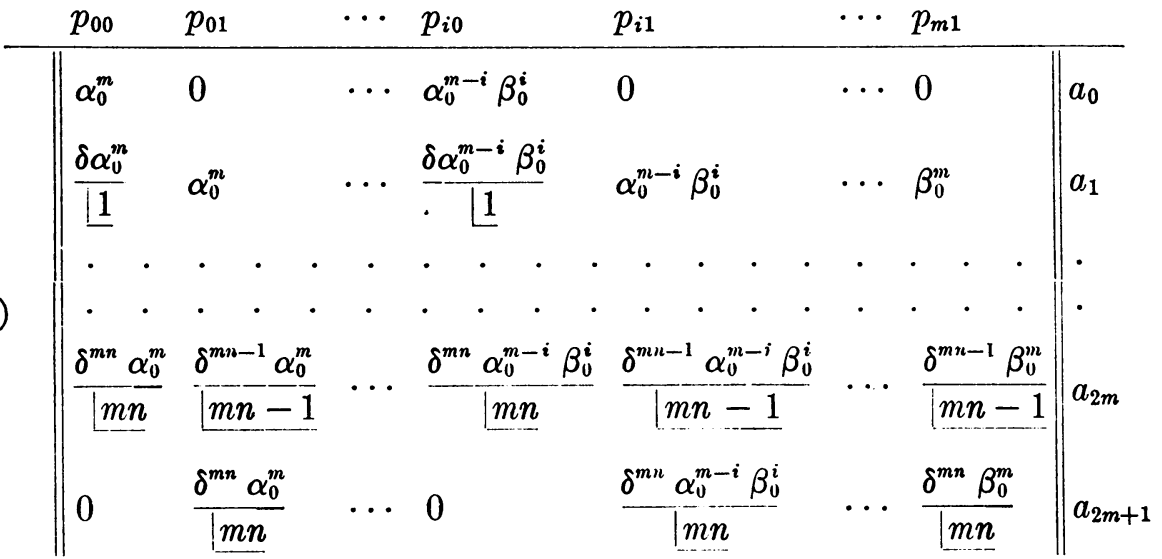

where the column on the right is the column of absolute terms, and

$$
\delta=2 \alpha_{1} \frac{\partial}{\partial \alpha_{0}}+\alpha_{2} \frac{\partial}{\partial \alpha_{1}}+2 \beta_{1} \frac{\partial}{\partial \beta_{0}}+\beta_{2} \frac{\partial}{\partial \beta_{1}} .
$$

\footnotetext{
* White, American Journal of Mathematics, vol. 17 (1895), p. 235.
} 
If we operate upon the determinant $D$ of this matrix by either one of the operators

$$
\left(\beta \frac{\partial}{\partial \alpha}\right),\left(\alpha \frac{\partial}{\partial \beta}\right)
$$

the result is a sum of $2 m+2$ determinants each having two columns alike and therefore zero. For, evidently,

$$
\begin{aligned}
& \left(\beta \frac{\partial}{\partial \alpha}\right) f_{12}^{m-i} f_{22}^{i}=(m-i) f_{12}^{m-i-1} f_{22}^{i+1}, \\
& \left(\alpha \frac{\partial}{\partial \beta}\right) f_{12}^{m-i} f_{22}^{i}=i f_{12}^{m-i+1} f_{22}^{i-1} \quad(i=0, \cdots, m) .
\end{aligned}
$$

Hence $D$ is a combinant of $f_{12}, f_{22}$, and, as the fundamental system of combinants of two quadratics contains only one invariant, their resultant $R$, we have $D=k R^{\frac{1}{2} m(m+1)}, k$ being numerical.

For similar reasons the determinants of the augmented matrix corresponding to the solution by Cramer's rule for the respective quantities

$$
p_{00}, p_{10}, \cdots, p_{r 0}, \cdots, p_{m 0},
$$

are all derivable from that corresponding to $p_{00}$ by successive operation by $-[\alpha(\partial / \partial \beta)]$ upon the latter. Also, successive operation by $-[\alpha(\partial / \partial \beta)]$ upon the determinant of the augmented matrix corresponding to the solution for $p_{01}$ gives the determinants of the augmented matrix corresponding to

$$
p_{01}, p_{11}, \cdots, p_{i 1}, \cdots, p_{m 1} \text {. }
$$

A similar type of expansion of a binary form $f_{m}$ of order $m$, with arbitrary coefficients, of which we shall make use, is one in terms of argument forms of unlike orders, $f_{1 n_{1}}, f_{2 n_{2}}$. We prove the following

Lemma. If $f_{1 n_{1}}, f_{2 r_{2}}$ are any two given binary quantics of respective orders $n_{1}, n_{2}$, and if

$$
\phi_{i m-n_{i}}=p_{i 0} x_{1}^{m-n_{i}}+p_{i 1} x_{1}^{m-n_{i}-1} x_{2}+\cdots+p_{i m-n_{i}} x_{2}^{m-n_{i}} \quad(i=1,2),
$$

then a necessary and sufficient condition in order that the linear system in the quantities $p_{i j}$ obtained by making

$$
f_{m}=\phi_{1 m-n_{1}} f_{1 n_{1}}+\phi_{2 m-n_{2}} f_{2 n_{2}} \quad\left(n_{1}+n_{2} \leqq m+1\right),
$$

identically, should be consistent, is that the resultant $R$ of $f_{1 n_{1}}$ and $f_{2 n_{2}}$ should be different from zero.

To prove this we assume

$$
f_{1 n_{1}}=\alpha_{0} x_{1}^{n_{1}}+\alpha_{1} x_{1}^{n_{1}-1} x_{2}+\cdots, \quad f_{2 n_{2}}=\beta_{0} x_{1}^{n_{2}}+\beta_{1} x_{1}^{n_{2}-1} x_{2}+\cdots,
$$


and, as $R \neq 0$ precludes $\alpha_{0}=0, \beta_{0}=0$ simultaneously, we let $\alpha_{0} \neq 0$. The matrix of the linear system is now

\begin{tabular}{|c|c|c|c|c|c|c|c|c|c|}
\hline \multicolumn{2}{|r|}{$p_{10}$} & \multicolumn{2}{|l|}{$p_{11}$} & \multirow{2}{*}{$\frac{p_{1 m-n_{1}}}{0}$} & \multirow{2}{*}{$\frac{p_{20}}{\beta_{0}}$} & \multirow{2}{*}{$\frac{p_{21}}{0}$} & \multicolumn{3}{|c|}{$p_{2 m-n_{2}}$} \\
\hline & $\alpha_{0}$ & 0 & $\cdots$ & & & & $\cdots$ & 0 & $a_{0}$ \\
\hline & $\alpha_{1}$ & $\alpha_{0}$ & $\cdots$ & 0 & $\beta_{1}$ & $\beta_{0}$ & $\ldots$ & 0 & $a_{1}$ \\
\hline & $\cdot$ & $\cdot$ & & $\cdot \cdot$ & . & & & $\cdot$ & $\cdot$ \\
\hline$M:$ & $\alpha_{n_{1}}$ & $\alpha_{n_{1}-1}$ & $\cdots$ & $\cdots$ & $\beta_{n_{2}}$ & $\beta_{n_{2}-1}$ & $\cdots$ & $\ldots$ & • \\
\hline & 0 & $\alpha_{n_{1}}$ & $\cdots$ & $\ldots$ & $\ldots$ & $\ldots$ & $\cdots$ & $\ldots$ & • \\
\hline & . & $0^{\circ}$ & & $\cdot \cdot$ & ${ }^{\cdot}{ }_{0}^{\circ}$ & · & & ${ }^{\cdot} \cdot$ & · \\
\hline
\end{tabular}

If we delete the first $\sigma=m+1-n_{1}-n_{2}$ columns of $\beta$ 's from $M$ and apply to the resulting square matrix $M^{\prime}$ properly chosen elementary transformations we get a square matrix in which the first $\sigma$ elements in the principal diagonal are $\alpha_{0}$ and all other elements in the first $\sigma$ rows and first $\sigma$ columns are zero. Then the complementary minor of the minor of order $\sigma$ in the upper left-hand corner of $M^{\prime}$ is the dialytic form of the resultant $R$, and, if $D$ is the determinant of $M^{\prime}, D=\alpha_{0}^{\sigma} R$. Hence if $R \neq 0$ the rank of $M$ is $m+1$, and the lemma is proved.*

If $n_{1}+n_{2}=m+1$, then $\sigma=0$, and the expansion (3) is unique.

Again if the order $m-n_{i}$ of $\phi_{i m-n_{i}}(i=1,2)$ is sufficiently large, in fact if $m-n_{i}+1 \geqq n_{1}+n_{2}$, the form $\phi_{i m-n_{i}}$ may itself be developed in an expansion (3) in terms of the argument forms $f_{1 n_{1}}, f_{2 n_{2}}$,

$$
\phi_{i m-n_{i}}=\phi_{1 m-n_{i}-n_{1}}^{\prime} f_{1 n_{1}}+\phi_{2 m-n_{i}-n_{2}}^{\prime} f_{2 n_{2}},
$$

and, so far as the orders permit repetitions, $\nu$ repetitions of this process for an arbitrary order $m$ gives an expansion more explicit than (3), viz.,

$$
f_{m}=\phi_{0}^{(\nu)} f_{1 n_{1}}^{a}+\phi_{1}^{(\nu)} f_{1 n_{1}}^{a-1} f_{2 n_{2}}+\cdots+\psi_{1}^{(\nu)} f_{1 n_{1}} f_{2 n_{2}}^{\beta-1}+\psi_{0}^{(\nu)} f_{2 n_{2}}^{\beta},
$$

wherein the coefficient forms are all of order less than $n_{1}+n_{2}-1$. This expansion is not unique, in general, for, as seen above, arbitrary parameters remain in the coefficients of the forms $\phi^{(\nu)}, \psi^{(\nu)}$, except in particular cases analogous to the case $n_{1}+n_{2}==m+1$ in (3).

2. Covariant expansions. Connection of the foregoing theory with invariant principles is made by regarding the argument forms in (1), (3), or (4) to be covariants of a definite set of linear transformations, either universal covariants of this set or covariants of $f$ under the set. The following existence theorem proved for expansions of type (3) holds as well for type (1) and the unique cases of (4).

* Bôcher, Introduction to Higher Algebra (1915), p. 46.

Trans. A m. Math. Soc. 29 
THEOREM. If $f_{1 n_{1}}, f_{2 n_{2}}$ are covariants under a definite transformation or group $G$, and if $n_{1}+n_{2}=m+1$ so that the expansion is unique, then the forms $\phi_{1 m-n_{1}}, \phi_{2 m-n_{2}}$ are covariants of $f$ under $G$.

For proof let (3) be written in the form

$$
f_{m}=\phi_{1}\left(A_{0}, A_{1}, \cdots ; x_{1}, x_{2}\right) f_{1 n_{1}}+\phi_{2}\left(A_{0}, A_{1}, \cdots ; x_{1}, x_{2}\right) f_{2 n_{2}},
$$

where $A_{j}$ is the rational function of $a_{0}, a_{1}, \cdots$ obtained by solving (3) for $p_{1 j}\left(j=0, \cdots, m-n_{1}\right)$ and $\mathrm{A}_{j}$ is the similar function equal to $p_{2 j}(j=0$, $\left.\cdots, m-n_{2}\right)$. Let $f_{m}$ be transformed by the general transformation of $G$, viz.,

$$
t:\left\{\begin{array}{l}
x_{1}=\alpha_{1} x_{1}^{\prime}+\alpha_{2} x_{2}^{\prime}, \\
x_{2}=\beta_{0} x_{1}^{\prime}+\beta_{1} x_{2}^{\prime} \quad\left(\alpha_{1} \beta_{1}-\alpha_{2} \beta_{0} \neq 0\right),
\end{array}\right.
$$

and suppose $f_{m}^{\prime}$ is expanded in terms of $f_{1 n_{1}}\left(x_{1}^{\prime}, x_{2}^{\prime}\right), f_{2 n 2}\left(x_{1}^{\prime}, x_{2}^{\prime}\right)$ by formula (3). Then

$$
f_{m}^{\prime}=\phi_{1}\left(A_{0}^{\prime}, A_{1}^{\prime}, \cdots ; x_{1}^{\prime}, x_{2}^{\prime}\right) f_{1 n_{1}}^{\prime}+\phi_{2}\left(\mathrm{~A}_{0}^{\prime}, \mathrm{A}_{1}^{\prime}, \cdots ; x_{1}^{\prime}, x_{2}^{\prime}\right) f_{2 n_{2}}^{\prime},
$$

where $A_{j}^{\prime}, \mathrm{A}_{j}^{\prime}$ are the same functions of $a_{0}^{\prime}, a_{1}^{\prime}, \cdots$ that $A_{j}, \mathrm{~A}$, respectively are of $a_{0}, a_{1}, \cdots$. Now if the inverse transformations on the $x$ 's and the $a$ 's are applied to all forms in equation (6) there results

(7) $f_{m}=\psi_{1}\left(B_{0}, B_{1}, \cdots ; x_{1}, x_{2}\right) c_{1} f_{1 n_{1}}+\psi_{2}\left(\mathrm{~B}_{0}, \mathrm{~B}_{1}, \cdots ; x_{1}, x_{2}\right) c_{2} f_{2 n_{2}}$,

in which $c_{1}, c_{2}$ are functions of the coefficients of the transformations $t$ only, such that

$$
f_{i}\left(x_{1}^{\prime}, x_{2}^{\prime}\right)=c_{i} f_{i}\left(x_{1}, x_{2}\right)
$$

Moreover $\psi_{1}=\phi_{1}\left(A_{0}^{\prime}, \cdots\right), \psi_{2}=\phi_{2}\left(\mathrm{~A}_{0}^{\prime}, \cdots\right)$ on account of the equalities of $t$. This expansion (7) must be identical with (3) since (3) is unique; and hence

$$
\begin{aligned}
& \phi_{1}\left(A_{1}^{\prime}, A_{0}^{\prime}, \cdots ; x_{1}^{\prime}, x_{2}^{\prime}\right)=c_{1}^{-1} \phi_{1}\left(A_{0}, A_{1}, \cdots ; x_{1}, x_{2}\right), \\
& \phi_{2}\left(\mathrm{~A}_{0}^{\prime}, \mathrm{A}_{1}^{\prime}, \cdots ; x_{1}^{\prime}, x_{2}^{\prime}\right)=c_{2}^{-1} \phi_{2}\left(\mathrm{~A}_{0}, \mathrm{~A}_{1}, \cdots ; x_{1}, x_{2}\right),
\end{aligned}
$$

which proves the theorem.

For expansion (1) the equations analogous to (8) are

(9) $\phi_{i n-1}\left(A_{i 0}^{\prime}, A_{i 1}^{\prime}, \cdots ; x_{1}^{\prime}, x_{2}^{\prime}\right)=c_{1}^{i-m} c_{2}^{-i} \phi_{i n-1}\left(A_{i 0}, A_{i 1}, \cdots ; x_{1}, x_{2}\right)$

$$
(i=0, \cdots, m) \text {. }
$$

In the case $n_{1}+n_{2}<m+1$ in (3) and for the non-unique cases of (4) the above proof does not hold. In numerous particular instances, however (cf. $\S$ III (26)), I have found that the arbitrary parameters which remain in the coefficient forms $\phi$ in these expansions can be determined so that relations analogous to

$$
\psi_{1}=\phi_{1}\left(A_{0}^{\prime}, \cdots\right), \quad \psi_{2}=\phi_{2}\left(\mathrm{~A}_{0}^{\prime}, \cdots\right),
$$


hold. The functions $\phi_{1}=\phi_{1 m-n_{1}}, \phi_{2}=\phi_{2 m-n_{2}}$ are then covariants whose coefficients involve arbitrary parameters.

3. Generalized typical representation. Let $S$ be any system of binary forms subject to general algebraic transformations $t$, and let $K$ be a complete system of concomitants of $S$, which contains two covariants $f_{1 n}, f_{2 n}$ of order $n$, whose resultant does not vanish.* Then every covariant $C$ of $S$ whose order is of the form $n(m+1)-1$ can be developed in a covariant expansion (1) and is thereby represented typically in terms of $f_{1 n}, f_{2 n}$ as arguments, and of other concomitants of $K$ of orders $<n$. The multiplier invariant in this representation is the resultant $R$ of $f_{1 n}, f_{2 n}$.

If $n=2, C$ represents any covariant of $S$ of odd order. Thus the concomitants in $K$ of orders $\leqq 2$ form an associated system for the representation of all covariants of odd order, in which the multiplier invariant is the resultant of any pair of quadratic covariants whose resultant does not vanish.

If we multiply any covariant $C$ of even order of $S$ by a definite linear covariant $l$ in $K$ and expand $l C$ in the arguments $f_{12}, f_{22}$ by formula (1) we get an expression of $C$ in terms of the covariants $f_{12}, f_{22}$ and concomitants of orders. $<2$ in which the denominator concomitant is of the form $l R^{a}$.

Again, covariants of $S$ can be represented typically in terms of two covariants of respective unlike orders $n_{1}, n_{2}$, when the orders of these covariants satisfy conditions which make expansion (3) or, more generally, expansion (4), unique. The multiplier invariant is then the resultant of the argument forms $f_{1 n_{1}}, f_{2 n_{2}}$.

The simplest system $S$ affording numerous examples of this theory is the well-known system of a quadratic $f$ and a cubic $g$. The fundamental set $K$ here contains the following fifteen forms: Invariants $D=(f, f)^{2},(f, \Delta)^{2}, R$, $\left(f^{3}, g^{2}\right)^{6},\left(f^{3}, g Q\right)^{6}, R$ being the discriminant of $g$; linear covariants $(f, g)^{2}$, $\left(f^{2}, g\right)^{3},(f, Q)^{2},\left(f^{2}, Q\right)^{3}$; quadratic covariants $f, \Delta=(g, g)^{2},(f, \Delta)^{1}$; cubic covariants $g,(f, g)^{1}, Q=(g, \Delta)^{1}$.

The problem of determining the associated system $A$ for orders $\leqq 2$ in this case requires only the selection of two of the three quadratic covariants as argument forms and expressing their resultant, the multiplier for the associated system, in terms of the invariants in $K$. All covariants in $K$ of orders greater than 2 are then expressed in terms of $A$ by expansion formula (1) (or (3) with $n_{1}=n_{2}=2$ ).

The resultant $\rho$ of any two quadratic forms is readily determined as the discriminant of their jacobian. Thus, writing $J=(\Delta, f)^{1}$,

$$
\frac{1}{2} \rho(\Delta, f)=\left((\Delta, f)^{1}, J\right)^{2}=\left((J, \Delta)^{1}, f\right)^{2} .
$$

\footnotetext{
* For a proof that two quadratic covariants with non-vanishing resultant exist for every form of even order $>4 \mathrm{cf}$. Clebsch, loc. cit., p. 410 .
} 
Applying the Gordan series

we have

$$
\left[\begin{array}{lll}
\Delta & f & \Delta \\
2 & 2 & 2 \\
0 & 1 & 1
\end{array}\right],
$$

Hence,

$$
(J, \Delta)^{1}=\frac{1}{2} R f-\frac{1}{2} \Delta(f, \Delta)^{2} .
$$

Similarly,

$$
\frac{1}{2} \rho(\Delta, f)=\frac{1}{2} R D-\frac{1}{2}\left[(f, \Delta)^{2}\right]^{2} .
$$

$$
\begin{aligned}
& \frac{1}{2} \rho\left((f, \Delta)^{1}, f\right)=\frac{1}{4} R D^{2}-\frac{1}{4} D\left[(f, \Delta)^{2}\right]^{2}, \\
& \frac{1}{2} \rho\left((f, \Delta)^{1}, \Delta\right)=\frac{1}{4} R^{2} D-\frac{1}{4} R\left[(f, \Delta)^{2}\right]^{2} .
\end{aligned}
$$

We append the resulting representation of the covariant $g$ when the arguments are chosen to be $\Delta, f$ :

$$
g=\frac{\frac{1}{2}\left[\left(f^{2}, Q\right)^{3}+\frac{1}{2}(f, g)^{2}(f, \Delta)^{2}\right] \Delta-\frac{1}{2} R(f, g)^{2} f}{-\left\{R D-\left[(f, \Delta)^{2}\right]^{2}\right\}} .
$$

In the case where $S$ consists of a single binary quintic $f$ the fundamental system $K$ consists of 23 concomitants, of which three are quadratics, viz.,

$$
i=(f, f)^{4},\left(i^{2}, H\right)^{4},\left(i^{3}, H\right)^{5},
$$

where $H$ is the hessian of $f$. Selecting the first two of these as argument forms, all covariants in $K$ of odd order $>2$, of which there are eight, are represented in terms of an associated set of orders $\leqq 2$. The hermitian skew invariant does not occur in the multiplier invariant $\rho$ as its degree is 18 , whereas the degree of $\rho$ is 16 .

\section{Systems belonging to PRescribed domains}

1. Domains of rationality. The general transformation $t$ leaves fixed a point $(k a, k b)$ provided that

$$
s=\left(\alpha_{1} s+\alpha_{2}\right) /\left(\beta_{0} s+\beta_{1}\right) \quad(s=a / b) .
$$

Thus $t$ leaves invariant the two linear forms

$$
f_{1}=2 \beta_{0} x_{1}+\left(\beta_{1}-\alpha_{1}+\Delta\right) x_{2}, \quad f_{-1}=2 \beta_{0} x_{1}+\left(\beta_{1}-\alpha_{1}-\Delta\right) x_{2},
$$

where $\Delta=\sqrt{ }\left(\beta_{1}-\alpha_{1}\right)^{2}+4 \beta_{0} \alpha_{2}$, the roots of $f_{1}, f_{-1}$ being the poles of $t$. Also $t$ has the covariant $f_{1} f_{-1}$, or

$$
J=\beta_{0} x_{1}^{2}+\left(\beta_{1}-\alpha_{1}\right) x_{1} x_{2}-\alpha_{2} x_{2}^{2} .
$$

Ordinary rational integral concomitants of a form under $t$, belong to a domain, which we designate by $R_{3}(1,0,0)$, determined by the coefficients and variables of that form. The covariant $J$ belongs to a domain obtained 
from $R_{3}$ by adjunction of the coefficients of the transformation $t$, which will be indicated by $R_{2}(1, t, 0)$. Moreover, $f_{1}$ and $f_{-1}$ belong to a domain $R_{1}(1, t, \Delta)$ obtained by adjunction of $\Delta$ to $R_{2}$.

The forms $f_{1}, f_{-1}, J$, and the concomitants considered below in this section, being functions of the coefficients of $t$, are primarily invariants of a single transformation $t$, but $\alpha_{1}$ and $\beta_{1}$ appear in them only in the combination $\alpha_{1}-\beta_{1}$; hence, if $\epsilon$ is a fixed constant, our invariants belong to a one-parameter continuous group leaving two points fixed, and characterized by the equation $\alpha_{1}-\beta_{1}=2 \epsilon \beta_{0}$. An important sub-problem under the general case is given by assuming

$$
\alpha_{1}-\beta_{1}=2 \epsilon \beta_{0}, \quad \alpha_{2}=-\beta_{0} .
$$

Then $J$, deprived of a constant factor, is a universal covariant of the set given by

$$
t_{1}: \begin{aligned}
& x_{1}=\left(2 \epsilon \beta_{0}+\beta_{1}\right) x_{1}^{\prime}-\beta_{0} x_{2}^{\prime} \\
& x_{2}=\beta_{0} x_{1}^{\prime}+\beta_{1} x_{2}^{\prime}
\end{aligned}
$$

of which the orthogonal transformations are a particular case. All of our systems, particularized for $t_{1}$, belong essentially to the domain $R_{3}(1,0,0)$.

Both orthogonal and boolean systems are special cases of the general systems given in this section. We shall revert to this phase of the question in Section III.

2. Complete systems in $R_{1}(1, t, \Delta)$ of the transformation $t$. If a binary form $f=a_{0} x_{1}^{m}+m a_{1} x_{1}^{m-1} x_{2}+\cdots$ be represented typically in terms of $f_{1}$, $f_{-1}$ as argument forms, the multiplier invariant, a power of which occurs in the denominator of the expansion of $f$, is $4 \beta_{0} \Delta$. This is an absolute constant and is, essentially, the quantity adjoined to define $R_{1}(1, t, \Delta)$. Writing the expansion in the form

$$
f=\phi_{m} f_{1}^{m}+m \phi_{m-2} f_{1}^{m-1} f_{-1}+\cdots+m \phi_{-(m-2)} f_{1} f_{-1}^{m-1}+\phi_{-m} f_{-1}^{m},
$$

we have in $\phi_{m-2 i}(i=0, \cdots, m)$ a set of $m+1$ linearly independent invariants of $t$ belonging to $R_{1}$, linear in the coefficients $a_{0}, \cdots, a_{m}$. The factor $r$ of $\phi_{m-2 i}$ in the equation expressing the invariant property

$$
\phi_{m-2 i}^{\prime}=r \phi_{m-2 i},
$$

is not a power of the determinant of $t,\left(\alpha_{1} \beta_{1}-\alpha_{2} \beta_{0}\right)$, but is a product of powers of the factors $\rho_{1}, \rho_{-1}$ of this determinant in $R_{1}$,

$$
\rho_{1}=\frac{1}{2}\left(\alpha_{1}+\beta_{1}+\Delta\right), \quad \rho_{-1}=\frac{1}{2}\left(\alpha_{1}+\beta_{1}-\Delta\right) .
$$

In fact, writing $c_{1}=\rho_{1}^{-1}, c_{2}=\rho_{-1}^{-1}$, it results from $I$ (9) that

$$
\begin{aligned}
\phi_{m-2 i}^{\prime} & =c_{1}^{i-m} c_{2}^{-i} \phi_{m-2 i} \\
& =\rho_{1}^{m-2 i}\left(\alpha_{1} \beta_{1}-\alpha_{2} \beta_{0}\right)^{i} \phi_{m-2 i} \quad(i=0, \cdots, m) .
\end{aligned}
$$


Moreover there follows the

Theorem. The $m+3$ forms $f_{1}, f_{-1}, \phi_{m-2 i}(i=0, \cdots \cdots, m)$ of the associated set constitute a fundamental system of concomitants of $f$ under $t$ in $R_{1}(1, t, \Delta)$.

The explicit systems in $R_{1}$ for $m=1,2,3$ are given below, in which, to secure isobarism, we write $\beta_{1}-\alpha_{1}=\gamma_{1}$.

$$
\begin{gathered}
\text { TABLE } \\
m=1 \\
2^{2} \beta_{0} \Delta \phi_{1}=\left(-\gamma_{1}+\Delta\right) a_{0}+2 \beta_{0} a_{1}, \\
-2^{2} \beta_{0} \Delta \phi_{-1}=\left(-\gamma_{1}-\Delta\right) a_{0}+2 \beta_{0} a_{1} . \\
m=2 .
\end{gathered}
$$

$2^{3} \beta_{0}^{2} \Delta^{2} \phi_{2}=\left(\gamma_{1}^{2}+2 \alpha_{2} \beta_{0}-\gamma_{1} \Delta\right) a_{0}+2 \beta_{0}\left(-\gamma_{1}+\Delta\right) a_{1}+2 \beta^{2} a_{2}$,

$2 \beta_{0} \Delta^{2} \phi_{0}=\alpha_{2} a_{0}+\gamma_{1} a_{1}-\beta_{0} a_{2}$,

$2^{3} \beta_{0}^{2} \Delta^{2} \phi_{-2}=\left(\gamma_{1}^{2}+2 \alpha_{2} \beta_{0}+\gamma_{1} \Delta\right) a_{0}+2 \beta_{0}\left(-\gamma_{1}-\Delta\right) a_{1}+2 \beta_{0}^{2} a_{2}$.

$$
m=3
$$

$-2^{4} \beta_{0}^{3} \Delta^{3} \phi_{3}=\left(\gamma_{1}^{3}+3 \beta_{0} \gamma_{1} \alpha_{2}-\left(\gamma_{1}^{2}+\beta_{0} \alpha_{2}\right) \Delta\right) a_{0}$

$$
+3 \beta_{0}\left(-\gamma_{1}^{2}-2 \beta_{0} \alpha_{2}+\gamma_{1} \Delta\right) a_{1}+3 \beta_{0}^{2}\left(\gamma_{1}-\Delta\right) a_{2}-2 \beta_{0}^{3} a_{3},
$$

$-2^{4} \beta_{0}^{3} \Delta^{3} \phi_{1}=\beta_{0} \alpha_{2}\left(\gamma_{1}-\Delta\right) a_{0}+\beta_{0}\left(\gamma_{1}^{2}-2 \beta_{0} \alpha_{2}-\gamma_{1} \Delta\right) a_{1}$

$$
+\beta_{0}^{2}\left(-3 \gamma_{1}+\Delta\right) a_{2}+2 \beta_{0}^{3} a_{3},
$$

$2^{4} \beta_{0}^{3} \Delta^{3} \phi_{-1}=\beta_{0} \alpha_{2}\left(\gamma_{1}+\Delta\right) a_{0}+\beta_{0}\left(\gamma_{1}^{2}-2 \beta_{0} \alpha_{2}+\gamma_{1} \Delta\right) a_{1}$

$2^{4} \beta_{0}^{3} \Delta^{3} \phi_{-3}=\left(\gamma_{1}^{3}+3 \beta_{0} \gamma_{1} \alpha_{2}+\left(\gamma_{1}^{2}+\beta_{0} \alpha_{2}\right) \Delta\right) a_{0}$

$+\beta_{0}^{2}\left(-3 \gamma_{1}-\Delta\right) a_{2}+2 \beta_{0}^{3} a_{3}$,

$$
+3 \beta_{0}\left(-\gamma_{1}^{2}-2 \beta_{0} \alpha_{2}-\gamma_{1} \Delta\right) a_{1}+3 \beta_{0}^{2}\left(\gamma_{1}+\Delta\right) a_{2}-2 \beta_{0}^{3} a_{3} \text {. }
$$

3. Systems belonging to $R_{2}(1, t, 0)$. It will be shown hereafter that complete systems of $f$ in $R_{2}$ may be constructed from associated systems by taking the set $S$ of ground forms to be the quantics $f, J$. Since, however, the forms $\phi_{m-2 i}$ do not satisfy some of the hypotheses (cf. (13)) fulfilled by ordinary invariants of $f$ under the group of general linear algebraic transformations, it is desirable to develop a special method for systems of $f$ under $t$ in $R_{2}$, and later to identify the results obtained by the two methods to be employed.

Concomitants in $R_{1}$ or $R_{2}$ may evidently be constructed by forming linear expressions $\psi$ in products of powers of $f_{1}, f_{-1}, \phi_{m-2 i}(i=0, \cdots, m) .^{*}$ A necessary, though not sufficient, condition in order that such an expression may be an invariant function in $R_{2}$ is that the exponent $\nu$ of $\rho_{1}$ (cf. (13)) in each and every term of the equation expressing invariancy should be 0 . The exponent $\nu$ for every term of any concomitant is equal to the sum of the

* Cf. Elliott, The syzygetic theory of orthogonal binariants, Proceedings of the London M athematical Society, vol. 33 (1901), p. 226. 
subscripts of the $\phi$ 's diminished by the exponent of $f_{1}$ and increased by that of $f_{-1}$ in any term. For pure invariants therefore,

$$
\nu=\sum_{i}(m-2 i) \text {. }
$$

If $d$ is the degree of such an invariant $\psi$ and if we revert to the notation for the $\phi$ 's used in I (1), $\phi_{m}=\phi_{00}, \phi_{m-2}=\phi_{10}, \cdots$, the sum $w$ of the (first) subscripts in each term of $\psi$ is $\sum i$. Thus

$$
\nu=d m-2 w, \quad w=\frac{1}{2}(d m-\nu) .
$$

We have thus established a one-to-one correspondence between invariants in the $\phi$ 's, of degree $d$ and factor $\rho_{1}^{\nu}$, and isobaric invariant functions of degree $d$ and weight $w$. The number of invariant expressions $\psi$ with factor $\rho_{1}^{\nu}$ is therefore the number of partitions of $w$ denoted by

$$
\left(\frac{1}{2}(d m-\nu) ; d, m\right)=\left(\frac{1}{2}(d m+\nu) ; d, m\right) .
$$

Now in the domain $R_{2}(1, t, 0)$ we are concerned with the case $\nu=0$. The actual invariant products of the $\phi$ 's for which $\nu=0$, each affected by that power of $D$ which is the multiplier in the invariant relation for the product, may be found from the caylean real generating function

$$
G(f)=\left\{\prod_{i=0}^{m}\left(1-\phi_{m-2 i} \rho_{1}^{m-2 i} D^{i}\right)\right\}^{-1} \quad\left(D=\alpha_{1} \beta_{1}-\alpha_{2} \beta_{0}\right) .
$$

The expansion of this function in ascending powers of $\rho_{1}$ has for the terms free from $\rho_{1}$ in it the sum of all of the products of the $\phi$ 's which are invariants with $\nu=0$, each product affected by the power of $D$ which is its multiplier. The invariants of this set which are asyzygetic are those possessing the same power of $D$ as multiplier.

Not all of these invariants belong to $R_{2}$. But the products may be arranged in conjugate pairs $\left(\psi_{p}, \psi_{-p}\right)$, etc., and by replacing $\psi_{p}, \psi_{-p}$ respectively by $\psi_{p}+\psi_{-p}, \psi_{p}-\psi_{-p}$ we get the complete asyzygetic sets which, neglecting factors involving $\Delta$, belong to $R_{2}$. In fact $\psi_{-p}$ is derived from $\psi_{p}$ by changing all factors $\phi_{m-2 i}$ into $\phi_{-(m-2 i)}$; and that the power of $D$ figuring as multiplier in the invariant relation for $\psi_{-p}$ is the same as the power for $\psi_{p}$ is easily proved. For, we have

$$
\psi_{p}=\phi_{m-2 i_{1}}^{p_{1}} \phi_{m-2 i_{2}}^{p_{2}} \cdots, \quad \psi_{-p}=\phi_{-\left(m-2 i_{1}\right)}^{p_{1}} \phi_{-\left(m-2 i_{2}\right)}^{p_{2}} \cdots .
$$

The multiplier for $\psi_{p}$ is $D^{p_{1} i_{1}+p_{2} i_{2}+\cdots}$, and that for $\psi_{-p}$ is $D^{p_{1}\left(m-i_{1}\right)+p_{2}\left(m-i_{2}\right)+\cdots}$. But if $\nu=\sum_{i}(m-2 i)=0$, then

$$
p_{1}\left(m-i_{1}\right)+p_{2}\left(m-i_{2}\right)+\cdots=p_{1} i_{1}+p_{2} i_{2}+\cdots .
$$

The generating function for simultaneous invariants is $\Pi G(f)$, the product 
including the $G(f)$ function for each form involved, and covariants of a single form are simultaneous invariants of the form and a linear quantic. Hence the generating function for covariants of $f$ in $R_{2}$ is

$$
\Gamma(f)=\left\{\prod_{i=0}^{m}\left(1-\phi_{m-2 i} \rho_{1}^{m-2 i} D^{i}\right)\left(1-f_{1} \rho_{1}^{-1}\right)\left(1-f_{-1} \rho_{1} D^{-1}\right)\right\}^{-1} .
$$

Thus, we have the

THEOREM. The products of functions $\phi_{m-2 i}$ occurring in the portion of the expansion of $\Gamma(f)$ free from $\rho_{1}$, which are affected by the factor $D^{k}$, furnish a complete asyzygetic set of concomitants in $R_{2}$ of multiplier $D^{k}$. These products have only to be arranged in conjugate pairs $\left(\psi_{p}, \psi_{-p}\right)$ and the terms of each pair replaced by $\psi_{p}+\psi_{-p}, \psi_{p}-\psi_{-p}$ respectively.

We shall now illustrate this theorem by exhibiting the explicit systems given by these methods for the forms of orders $1,2,3$.

$m=1$. The generating function for invariants of a linear form $f$ is

$$
G(f)=\left\{\left(1-\phi_{1} \rho_{1}\right)\left(1-\phi_{-1} \rho^{-1} D\right)\right\}^{-1} .
$$

When this is expanded it is found that the terms free from $\rho_{1}$ are

$$
1+\phi_{1} \phi_{-1} D+\phi_{1}^{2} \phi_{-1}^{2} D^{2}+\cdots \equiv\left(1-\phi_{1} \phi_{-1} D\right)^{-1} .
$$

Hence a linear form has only one invariant in $R_{2}$, viz., $\phi_{1} \phi_{-1}$ of index 1 . Moreover the terms free from $\rho_{1}$ in $G(f)$, when separated from the rest, are all given by the formula*

$$
1-\phi_{1} \phi_{-1} D
$$

This formula will be called the real generating function for invariants in the domain $R_{2}$. The form of the denominator of such a function shows what invariants should be selected to form a complete system in this domain.

The generating function for covariants and invariants both is

$$
\Gamma(f)=\left\{\left(1-\phi_{1} \rho_{1}\right)\left(1-\phi_{-1} \rho_{1}^{-1} D\right)\left(1-f_{1} \rho_{1}^{-1}\right)\left(1-f_{-1} \rho_{1} D^{-1}\right)\right\}^{-1} .
$$

Separation of the terms free from $\rho_{1}$ from $\Gamma(f)$ gives the terms furnished by the expansion of the following real generating function for concomitants of the linear form in $R_{2}$,

$$
\frac{1-\phi_{1} \phi_{-1} f_{1} f_{-1}}{\left(1-\phi_{1} \phi_{-1} D\right)\left(1-\phi_{1} f_{1}\right)\left(1-\phi_{-1} f_{-1}\right)\left(1-f_{1} f_{-1} D^{-1}\right)} .
$$

Accordingly the following four forms constitute a fundamental system of $f$ under $t$ in $R_{2}(1, t, 0)$ (cf. table for $m=1$ in the preceding paragraph):

* Cf. Elliott, Proceedings of the London Mathematical Society, vol. 33 (1901), p. 236. 


$$
\lambda=\phi_{1} \phi_{-1}, \quad \lambda_{1}=\phi_{1} f_{1}+\phi_{-1} f_{-1}, \quad \lambda_{-1}=\phi_{1} f_{1}-\phi_{-1} f_{-1},
$$

$$
\mu=f_{1} f_{-1} \text {, }
$$

where

$$
\begin{aligned}
I & =4 \beta_{0} \Delta^{2} \lambda=\alpha_{2} a_{0}^{2}+\gamma_{1} a_{0} a_{1}-\beta_{0} a_{1}^{2}, \\
C_{-1} & =\lambda_{-1}=\left(-\gamma_{1} a_{0}+2 \beta_{0} a_{1}\right) x_{1}+\left(2 \alpha_{2} a_{0}+\gamma_{1} a_{1}\right) x_{2}, \\
C_{1} & =\lambda_{1}=a_{0} x_{1}+a_{1} x_{2} \equiv f, \\
J & =\left(4 \beta_{0}\right)^{-1} \mu=\beta_{0} x_{1}^{2}+\gamma_{1} x_{1} x_{2}-\alpha_{2} x_{2}^{2} .
\end{aligned}
$$

These forms are connected by the syzygy

$$
S \equiv\left(4 \beta_{0} \alpha_{2}+\gamma_{1}^{2}\right) f^{2}-C_{-1}^{2}-4 I J=0 .
$$

$\boldsymbol{m}=2$. The generating function for concomitants of a quadratic $f$ is

$$
\Gamma(f)=\left\{\left(1-\phi_{2} \rho_{1}^{2}\right)\left(1-\phi_{0} D\right)\left(1-\phi_{-2} \rho_{1}^{-2} D^{2}\right)\right.
$$

$$
\left.\left(1-f_{1} \rho_{1}^{-1}\right)\left(1-f_{-1} \rho_{1} D^{-1}\right)\right\}^{-1} \text {. }
$$

The real generating function for concomitants in $R_{2}$ is found to be

$$
\frac{1-\phi_{2} \phi_{-2} f_{1}^{2} f_{-1}^{2}}{\left(1-\phi_{0} D\right)\left(1-\phi_{2} \phi_{-2} D^{2}\right)\left(1-\phi_{2} f_{1}^{2}\right)\left(1-\phi_{-2} f_{-1}^{2}\right)\left(1-f_{1} f_{-1} D^{-1}\right)} \cdot
$$

Hence the fundamental system of $f$ in $R_{2}$ consisis of the following five forms from which extraneous factors involving $\Delta$ are to be deleted:

$$
\begin{gathered}
\lambda_{0}=\phi_{0}, \quad \lambda=\phi_{2} \phi_{-2}, \quad \lambda_{1}=\phi_{2} f_{1}^{2}+\phi_{-2} f_{-1}^{2}, \\
\lambda_{-1}=\phi_{2} f_{1}^{2}-\phi_{-2} f_{-1}^{2}, \quad \mu=f_{1} f_{-1} .
\end{gathered}
$$

This system is given in explicit form below:*

$$
\begin{aligned}
& I_{0}=2 \beta_{0} \Delta^{2} \lambda_{0}=\alpha_{2} a_{0}+\gamma_{1} a_{1}-\beta_{0} a_{2}, \\
& I=16 \beta_{0}^{2} \Delta^{4} \lambda=\alpha_{2}^{2} a_{0}^{2}-4 \alpha_{2} \beta_{0} a_{1}^{2}+\beta_{0}^{2} a_{2}^{2}+2 \alpha_{2} \gamma_{1} a_{0} a_{1} \\
& +\left(\gamma_{1}^{2}+2 \alpha_{2} \beta_{0}\right) a_{0} a_{2}-2 \beta_{0} \gamma_{1} a_{1} a_{2}, \\
& C_{1}=\Delta^{2} \lambda_{1}=\left(\left(\gamma_{1}^{2}+2 \alpha_{2} \beta_{0}\right) a_{0}-2 \beta_{0} \gamma_{1} a_{1}+2 \beta_{0}^{2} a_{2}\right) x_{1}^{2} \\
& +2\left(-\alpha_{2} \gamma_{1} a_{0}+4 \alpha_{2} \beta_{0} a_{1}+\beta_{0} \gamma_{1} a_{2}\right) x_{1} x_{2} \\
& +\left(2 \alpha_{2}^{2} a_{0}+2 \alpha_{2} \gamma_{1} a_{1}+\left(\gamma_{1}^{2}+2 \alpha_{2} \beta_{0}\right) a_{2}\right) x_{2}^{2} \text {, } \\
& C_{-1}=\Delta \lambda_{-1}=\left(-\gamma_{1} a_{0}+2 \beta_{0} a_{1}\right) x_{1}^{2}+2\left(\alpha_{2} a_{0}+\beta_{0} a_{2}\right) x_{1} x_{2} \\
& J=\left(4 \beta_{0}\right)^{-1} \mu=\beta_{0} x_{1}^{2}+\gamma_{1} x_{1} x_{2}-\alpha_{2} x_{2}^{2} . \\
& +\left(2 \alpha_{2} a_{1}+\gamma_{1} a_{2}\right) x_{2}^{2},
\end{aligned}
$$

* In place of $C_{1}$ we may use $f$ since $\Delta^{2} f=C_{1}+2 I_{0} J$. 
A single syzygy connects these forms, viz.,

$$
S \equiv 4 I J^{2}-C_{1}^{2}+\left(4 \beta_{0} \alpha_{2}+\gamma_{1}^{2}\right) C_{-1}^{2}=0 .
$$

$\boldsymbol{m}=3$. In a similar manner, employing the real generating function for concomitants of the cubic in $R_{2}$, we derive as a fundamental system the following thirteen quantics (cf. table in II 2):

$$
\begin{gathered}
\phi_{3} \phi_{-3}, \quad \phi_{1} \phi_{-1}, \phi_{3} \phi_{-1}^{3}+\phi_{-3} \phi_{1}^{3}, \quad \phi_{3} \phi_{-1}^{3}-\phi_{-3} \phi_{1}^{3}, f_{1} f_{-1}, \\
\phi_{1} f_{1} \pm \phi_{-1} f_{-1}, \quad \phi_{3} f_{1}^{3} \pm \phi_{-3} f_{-1}^{3}, \\
\phi_{3} \phi_{-1} f_{1}^{2} \pm \phi_{-3} \phi_{1} f_{-1}^{2}, \quad \phi_{3} \phi_{-1}^{2} f_{1} \pm \phi_{-3} \phi_{1}^{2} f_{-1} .
\end{gathered}
$$

\section{INVARIANT SYSTEMS FOR SPECIAL GROUPS}

1. Complete systems derived from associated systems. The systems treated in section II may be derived from certain associated systems, and by a method which is of importance in the invariant theory of various special sets of transformations.

Construct; in terms of the forms $f_{1}, f_{-1}$ as arguments, the typical representation of the system $S$ of ground forms consisting of an mic $f$ and the covariant $J$. The representation of $f$ is given by (11), that of $J$ is $J$ itself since $f_{1} f_{-1}=4 \beta_{0} J$. The multiplier of the associated system is the absolute constant $4 \beta_{0} \Delta$ which we adjoin to the domain. The fundamental system of $f$ under $t$ in $R_{2}$ then consists of an associated system of $S=\{f, J\}$, with $\Delta$, as a constant, omitted. We can construct this associated set explicitly by making the following substitution in the associated system of an mic $f$ and a quadratic $J_{1}=b_{0} x_{1}^{2}+2 b_{1} x_{1} x_{2}+b_{2} x_{2}^{2}$, in which the multiplier invariant is the square root of the discriminant of $J_{1}$ :

$$
\left(\begin{array}{llllll}
a_{i} & b_{0} & b_{1} & b_{2} & x_{1} & x_{2} \\
\phi_{m-2 i} & 0 & 1 & 0 & f_{1} & f_{-1}
\end{array}\right) \quad(i=0, \cdots, m) .
$$

Thus, in case $m=2$, the covariant

$$
\left(a_{0} b_{1}-a_{1} b_{0}\right) x_{1}^{2}+\left(a_{0} b_{2}-a_{2} b_{0}\right) x_{1} x_{2}+\left(a_{1} b_{2}-a_{2} b_{1}\right) x_{2}^{2}
$$

becomes

$$
\phi_{2} f_{1}^{2}-\phi_{-2} f_{-1}^{2} \text {, }
$$

and this is the fourth form of $\left(17_{1}\right)$. Similarly the sets $\left(16_{1}\right),\left(17_{1}\right),(18)$ may all be derived from the corresponding associated systems of $f$ and $J_{1}$.

2. Orthogonal systems. An obscurity which has long persisted in the theory of orthogonal invariants may now be cleared up. To quote from the paper by Elliott on orthogonal binariants referred to above: "Absolute orthogonal invariants of a $p$-ic are invariants of the $p$-ic and $x_{1}^{2}+x_{2}^{2}$ and 
vice versa. It has therefore been hastily supposed elsewhere that the complete irreducible invariant system of a $p$-ic and a quadratic produces exactly the complete (absolute) orthogonal system for the $p$-ic, when $x_{1}^{2}+x_{2}^{2}$ is taken for the quadratic. A first case of the redundancy of the former system for the latter purpose is exhibited above [the reducibility of the covariant $Q$ of the cubic in terms of the orthogonal system corresponding to the set (18)]. The search for complete absolute orthogonal systems is not identical with the search for invariant systems of forms one of which is a quadratic."

The inference here is that orthogonal systems require a special method of treatment not comprehended in the ordinary theory for integrally complete systems under the general binary group $G$. We can now show, however, that the orthogonal invariant theory is essentially identical with the theory of associated systems under $G$. For, by particularizing the transformation $t_{1}$ of II 1 into the orthogonal transformations

$$
x_{1}=x_{1}^{\prime} \cos \theta-x_{2}^{\prime} \sin \theta, \quad x_{2}=x_{1}^{\prime} \sin \theta+x_{2}^{\prime} \cos \theta
$$

(making $\left.\epsilon=0, \beta_{0}=\sin \theta, \beta_{1}=\cos \theta\right) J$ becomes essentially $x_{1}^{2}+x_{2}^{2}$, while the concomitants exemplified in the table in II 2 are freed from the coefficients of the transformation and become the non-absolute orthogonal concomitants of the respective forms $f$, the multiplier $\Delta$ of the associated system now becoming a constant times $\sqrt{-1}$. At the same time the sets in $R_{2},\left(16_{1}\right)$, $\left(17_{1}\right),(18)$ are also freed from the coefficients of the transformation and become the complete absolute orthogonal systems.

Thus the absolute orthogonal invariant system of a form $f$ is given precisely by the associated system of the set $f, x_{1}^{2}+x_{2}^{2}$, where the multiplier invariant is the square root of the discriminant of $x_{1}^{2}+x_{2}^{2}$.

The lowest instance where this associated set is smaller in number than the ordinary integrally complete simultaneous system of $f$ and $x_{1}^{2}+x_{2}^{2}$ is where $m=3$. In fact, in the general case of the system (18) of the transformation $t$, the covariant $Q$ of the cubic is reducible, on multiplication by a power of $\Delta$, in terms of the set (18).

3. Boolean systems. If we particularize $t_{1}$ of II 1 by the substitutions

$$
\beta_{0}=\frac{\sin \alpha}{\sin w}, \quad \beta_{1}=\frac{\sin \beta}{\sin w}, \quad \epsilon=-\cos w,
$$

and assume $w=\beta-\alpha$, we get the following transformations of determinant unity,

$$
x_{1}=x_{1}^{\prime} \frac{\sin (w-\alpha)}{\sin w}+x_{2}^{\prime} \frac{\sin (w-\beta)}{\sin w}, \quad x_{2}=x_{1}^{\prime} \frac{\sin \alpha}{\sin w}+x_{2}^{\prime} \frac{\sin \beta}{\sin w},
$$

representing rotation of a pair of oblique coördinate axes inclined at an angle $w$ 
into another pair inclined at the same angle. Then $J$ becomes a constant times the universal covariant (quâ transformations on $x_{1}, x_{2}$ and not on $w$ )

$$
q=x_{1}^{2}+2 \cos w x_{1} x_{2}+x_{2}^{2} .
$$

Hence if we make the substitutions (20) in the systems $\left(16_{1}\right),\left(17_{1}\right),(18)$ these become the boolean systems of the forms of respective orders $1,2,3$.

Moreover, the invariants in the table in II 2 become, under (20), systems of a species of boolean invariant in which the factor in the invariant equation is a product of powers of $\rho_{1}, \rho_{-1}$ as below (cf. (13)),

$$
\rho_{1}=\cos \alpha+i \sin \alpha, \quad \rho_{-1}=\cos \alpha-i \sin \alpha .
$$

The two invariants of the linear form, for example, become

$$
(\cos w-i \sin w) a_{0}-a_{1}, \quad(\cos w+i \sin w) a_{0}-a_{1} .
$$

4. Kleinian groups. The formal nature of the invariant systems treated in section II shows that they may be interpreted when the transformation $t$ has complex coefficients $\alpha_{1}, \alpha_{2}, \beta_{0}, \beta_{1}$. In particular, the multiply infinite set represented by

$$
t_{1}: \begin{aligned}
& x_{1}=\left(2 \epsilon \beta_{0}+\beta_{1}\right) x_{1}^{\prime}-\beta_{0} x_{2}^{\prime}, \\
& x_{2}=\beta_{0} x_{1}^{\prime}+\beta_{1} x_{2}^{\prime},
\end{aligned}
$$

where $\epsilon$ is a fixed and $\beta_{0}, \beta_{1}$ arbitrary complex numbers, has the universal covariant

$$
q=x_{1}^{2}-2 \epsilon x_{1} x_{2}+x_{2}^{2}
$$

and the systems tabulated in II, particularized for $t_{1}$, become complete systems with complex coefficients, free from the arbitrary coefficients in $t_{1}$. Note that the latter transformation includes all types of substitutions with complex coefficients excepting the parabolic type, i. e., includes the elliptic, hyperbolic, and the loxodromic types.*

5. The general cyclic group on $n$ variables. The following transformations generate the so-called cyclic group $C$ on $n$ variables:

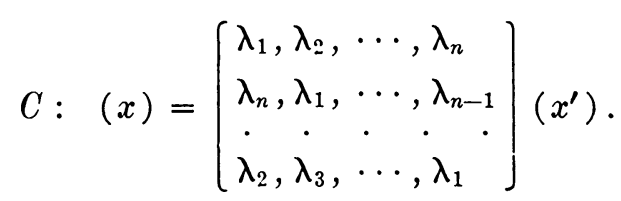

The determinant $D$ of $C$ is a linearly factorable $n$-ary form of order $n$. For, let us multiply its columns by $1, g^{t}, \cdots, g^{(n-1) t}$, respectively, where $g$ is an arbitrary primitive $n$th root of unity, and $t \leqq n-1$, and add all to the first

\footnotetext{
* Klein-Fricke, Theorie der elliptischen Modulfunctionen (1890), p. 163.
} 
column. The result is immediately of the form

where

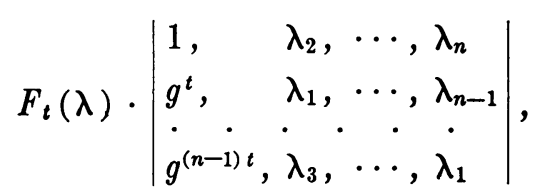

and hence

$$
F_{t}(\lambda)=\lambda_{1}+g^{t} \lambda_{2}+g^{2 t} \lambda_{3}+\cdots+g^{(n-1) t} \lambda_{n}
$$

$$
D=\prod_{t=0}^{n-1} F_{t}(\lambda)
$$

The following theorem is easily proved:

Theorem. The forms $F_{t}(x)(t=0, \cdots, n-1)$ are universal covariants of $C$, satisfying the invariant relations

$$
F_{t}\left(x^{\prime}\right)=F_{(n-1) t}(\lambda)^{-1} F_{t}(x) .
$$

The general quantic $f$ of order $m$ in $n$ variables, whose coefficients are arbitrary variables, can be given a typical representation by expanding it in terms of the forms $F_{t}(x)(t=0, \cdots, n-1)$ as arguments. The multiplier of the resulting associated system, i. e., the invariant, a power of which occurs in the denominator in the expansion of $f$, is the eliminant of the $n$ linear arguments. ${ }^{*}$ This resultant, being a function of $n$th roots of unity only, is a constant which we may adjoin to the domain of rationality. The coefficients in the expansion will be linear expressions in the $\left(\begin{array}{c}m+n-1 \\ m\end{array}\right)$ coefficients of $f$, belonging to the domain $R$ of rational functions of the $n$th roots of unity. These linear expressions, $\left(\begin{array}{c}m+n-1 \\ m\end{array}\right)$ in number, constitute a complete system of invariants of $f$ under $C$ in $R$. $\dagger$

6. A transformation leaving an involution fixed. The systems in $R_{2}$ treated in section II are complete for any transformation $t$ whose only covariant in $R_{2}$ is a power of the quadratic represented by $J$. There exist no covariants of the general transformation $t$ other than $f_{1} f_{-1}$ and its powers. This appears by consideration of the generating functions employed in II, but a direct proof of the fact is possible. Since $t$ has but two poles, any other quadratic covariant of $t$, as

$$
K=x_{1}^{2}-2 b x_{1} x_{2}+c x_{2}^{2},
$$

must have its roots interchanged by $t$. But the condition

$$
b \mp \sqrt{b^{2}-c}=\frac{\alpha_{1}\left(b \pm \sqrt{b^{2}-c}\right)+\alpha_{2}}{\beta_{0}\left(b \pm \sqrt{b^{2}-c}\right)+\beta_{1}}
$$

gives $\beta_{1}=-\alpha_{1}$.

* Compare a paper by the present writer, these Transactions, vol. 15 (1914), p. 83 .

† For a treatment of the case $n=3$, cf. Elliott, Messenger of $\mathrm{M}$ a the matics, vol. 33 (1903-4), p. 108. 
Under the transformation with arbitrary coefficients,

$$
T: x_{1}=\alpha_{1} x_{1}^{\prime}+\alpha_{2} x_{2}^{\prime}, \quad x_{2}=\beta_{0} x_{1}^{\prime}-\alpha_{1} x_{2}^{\prime},
$$

the following quadratic forms are relative covariants:

$$
f_{12}=\beta_{0} x_{1}^{2}+\alpha_{2} x_{2}^{2}, \quad f_{22}=\alpha_{1} x_{1}^{2}+\alpha_{2} x_{1} x_{2} .
$$

These forms are left invariant by having their roots interchanged, and all quadratics thus left invariant belong to the involution

In fact, equation (24) gives

$$
L=k_{1} f_{12}+k_{2} f_{22} \text {. }
$$

$$
\beta_{0} c-2 \alpha_{1} b-\alpha_{2}=0,
$$

and hence the following equality is an identity:

$$
K=\frac{2 \alpha_{1} b+\alpha_{2}}{\beta_{0} \alpha_{2}} f_{12}-\frac{2 b}{\alpha_{2}} f_{22} .
$$

The covariant $J=\beta_{0} x_{1}^{2}-2 \alpha_{1} x_{1} x_{2}-\alpha_{2} x_{2}^{2}$ is the jacobian of $f_{12}, f_{22}$. Hence the

TheOREM. A necessary and sufficient condition in order that a binary form be transformed into itself by $T$ is that it be equal to a power of $J$ times a product of powers of forms in the involution $L$. The double points of this involution are the poles of $T$.

If we expand a quantic $f_{2 m+1}$ in the argument forms $f_{12}, f_{22}$ according to the formula (1) $\mathrm{I}$, the coefficient forms $\phi_{i 1}(i=0, \cdots, m)$ are linear covariants of $f_{2 m+1}$ under $T$ in $R_{2}(1, T, 0)$. Then it is manifest that a fundamental system of concomitants of $f_{2 m+1}$ under $T$ in this domain is precisely the simultaneous system of the $m+1$ linear covariants $\phi_{i 1}(i=0, \cdots, m)$ taken with the covariants of the transformation, e. g., $f_{12}, f_{22}$. Definitive processes appertaining to $T$ for the construction of this joint system are not known.

We append the coefficient covariants $\phi_{01}, \phi_{11}$ for the case where the expanded form is a cubic. If $D=-\alpha_{1}^{2}-\beta_{0} \alpha_{2}$, then,

$$
\begin{aligned}
-\alpha_{2} D \phi_{01} & =\left(\alpha_{2}^{2} a_{0}-3 \alpha_{1} \alpha_{2} a_{1}+3 \alpha_{1}^{2} a_{2}+\alpha_{1} \beta_{0} a_{3}\right) x_{1}-D a_{3} x_{2}, \\
-\alpha_{2} D \phi_{11} & =\left(\alpha_{1} \alpha_{2} a_{0}+3 \alpha_{2} \beta_{0} a_{1}-3 \alpha_{1} \beta_{0} a_{2}-\beta_{0}^{2} a_{3}\right) x_{1} \\
& \quad+\left(-\alpha_{2}^{2} a_{0}+3 \alpha_{1} \alpha_{2} a_{1}+3 \alpha_{2} \beta_{0} a_{2}-\alpha_{1} \beta_{0} a_{3}\right) x_{2} .
\end{aligned}
$$

Note that the so-called skew-orthogonal transformations form a special case of $T$, but, also, that in this case the poles are real and rational, and the domains $R_{1}, R_{2}$ coincide.

7. Modular systems. One of the main features of the general theory of 
sections I, II of this paper is its character as a formal algorithm under which a variety of invariant systems for special groups can be studied.

Consider the group of binary transformations $T$ in which the four parameters are least residues modulo $p$, where $p$ is a prime number. A complete system of universal covariants $\bmod p$ of $T$ consists of*

$L=x_{1}^{p} x_{2}-x_{1} x_{2}^{p}, \quad Q=\left(x_{1}^{p^{2}} x_{2}-x_{1} x_{2}^{p^{2}}\right) \div L=x_{1}^{p(p-1)}+\cdots+x_{2}^{p(p-1)}$.

Regarding all equations in I 2 to be congruences modulo $p$, and setting $f_{1 n_{1}}=Q, f_{2 n_{2}}=L$ in (3) and in (4), it appears that a binary form of order $m=p^{2}$ is reducible in terms of $Q$ and $L$ and two first degree formal modular covariants of respective orders $p$ and $p^{2}-p-1$. This raises the question whether the modular expansions (3) for $m>p^{2}$, containing arbitrary parameters in their coefficients, may always have these parameters determined so the coefficient forms $\phi_{1 m-p^{2}+p}, \phi_{2 m-p-1}$ are modular covariants. Assuming this, (4) shows that the general quantic of order $m>p^{2}-1$ is reducible modulo $p$ in terms of $Q, L$, and its own first degree covariants of orders from 0 to $p^{2}-1$ inclusive.

For the case $p=2 \mathrm{I}$ have answered this question in the affirmative for the general order $m . \dagger$

For the case $p=3 \mathrm{I}$ have actually reduced in this manner the forms of orders $m=3^{2}, m=10, m=11$, thus verifying the existence of the desired expansion in a very typical case. Thus, while the theorem given below has not been proved in as general a form as is stated, I venture to give it in its general form for the notice of mathematicians who may be interested.

Since the proposition relates to the reducibility of the general quantic we may state it as a theorem on covariants.

Theonem. The maximum order of an irreducible covariant modulo $p$ of any binary form or set of forms is $p^{2}-1$.

For the case $m=11, p=3$, we find $f \equiv \phi_{15} Q+\phi_{27} L(\bmod 3)$, where

where

$$
\begin{aligned}
\phi_{15}= & a_{0} x_{1}^{5}+\left(a_{1}+a_{3}+\lambda S\right) x_{1}^{4} x_{2}+\left(a_{2}+a_{4}+a_{6}-\lambda T\right) x_{1}^{3} x_{2}^{2} \\
& +\left(a_{5}+a_{7}+a_{9}-\lambda S\right) x_{1}^{2} x_{2}^{3}+\left(a_{8}+a_{10}+\lambda T\right) x_{1} x_{2}^{4}+a_{11} x_{2}^{5},
\end{aligned}
$$

$$
S=a_{1}+a_{3}+a_{5}+a_{7}+a_{9}, \quad T=a_{2}+a_{4}+a_{6}+a_{8}+a_{10},
$$

and $\lambda$ is any least positive residue modulo 3 . The septic covariant $\phi_{27}$ is readily obtained as the quotient modulo 3 of $f-Q \phi_{15}$ divided by $L$.

A complete system of formal modular concomitants of a binary form $f$ could be obtained, under this theorem, as a simultaneous system of a set of

* Dickson, these Trans a c tion s, vol. 12 (1911), p. 75.

† O. E. Glenn, these Trans a ction s, vol. 17 (1916), p. 545. 
first degree covariants of $f$ of orders not exceeding $p^{2}-1$. Note, however, that the methods by which this simultaneous system would be constructed would be the definitive construction processes for simultaneous invariants and covariants under the modular group $T$, and these definitive construction processes are not fully known. Nevertheless, for fundamental systems of pure invariants in the modular realm, Dickson's theory of classes furnishes the requisite definitive methods.

University of Pennsyluania 